\title{
Processing of the Quench Detection Signals in W7-X
}

\author{
Dietrich Birus $^{1}$, Matthias Schneider ${ }^{1}$, Thomas Rummel ${ }^{1}$, Marko Fricke ${ }^{1}$, Klaus Petry ${ }^{2}$, Andreas \\ Ebersoldt $^{2}$ \\ ${ }^{1}$ Max-Planck-Institut für Plasmaphysik, Euratom Association \\ Teilinstitut Greifswald, Wendelsteinstr. 1, D-17491 Greifswald, Germany \\ ${ }^{2}$ Forschungszentrum Karlsruhe, Institut für Prozessdatendatenverarbeitung und Elektronik, Hermann- \\ von-Helmholtz-Platz 1, D-76344 Eggenstein-Leopoldshafen, Germany
}

\begin{abstract}
The Wendelstein 7-X (W7-X) project uses superconductive coils for generation of the magnetic field to keep the plasma. One of the important safety systems is the protection against quench events. The Quench Detection System of W7-X protects the superconducting coils, the superconducting bus bar sections and the high temperature superconductor of the current leads against the damage because of a quench and against the high stress by a fast discharge of the magnet system.

Therefore, the present design of the Quench Detection System (QDS) uses a two-stage safety concept for discharging the magnetic system. This paper describes the present design of the system assembly from the Quench Detection Unit (QDU) for the detection of the quench to the Quench Detection Interface (QDI) to implement the two-stage safety concept.
\end{abstract}

\section{Introduction}

The whole Quench Detection System consist of five QD subsystems, each with 72 QDU, an embedded PC for data storage and monitoring of the QDU and DC UPS, and two redundant Quench Detection Interfaces for the coupling to the magnet control system and a host control system. The QD Interface evaluates the quench and the failure information of the QD Subsystems in two safety levels and generates the control signal for the fast or the slow discharge of the magnet system.

The QDU is designed with an internal failsafe, programmable self test and redundancy feature with two independent detectors, broken wire check of the quench detection cables and connectors and two interfaces for transferring the measuring QD data to the embedded PC and to set up the QDU itself. 


\section{Quench Detection System}

Figure 1 illustrates the structurally design of the W7-X Quench Detection System. The QD subsystems are arranged around the W7-X torus. The two redundant QD interfaces are located in separately building as well as the host control.

The Quench Detection System supervises superconducting coils, the superconducting bus bar sections and the high temperature superconductor of the current leads against the damage because of a quench. Important properties of the W7-X Quench Detection System are:

- possibility to detect quenches at independent threshold values

- cable detection of the QD cables

- automatic self test of the QD units

- data recording of quench events while pre and post time

- discharging of the magnetic system at two safety levels

- remote controlling and parameterization of QD units and QD interfaces

- independent operating of QD subsystems using UPS and embedded PC

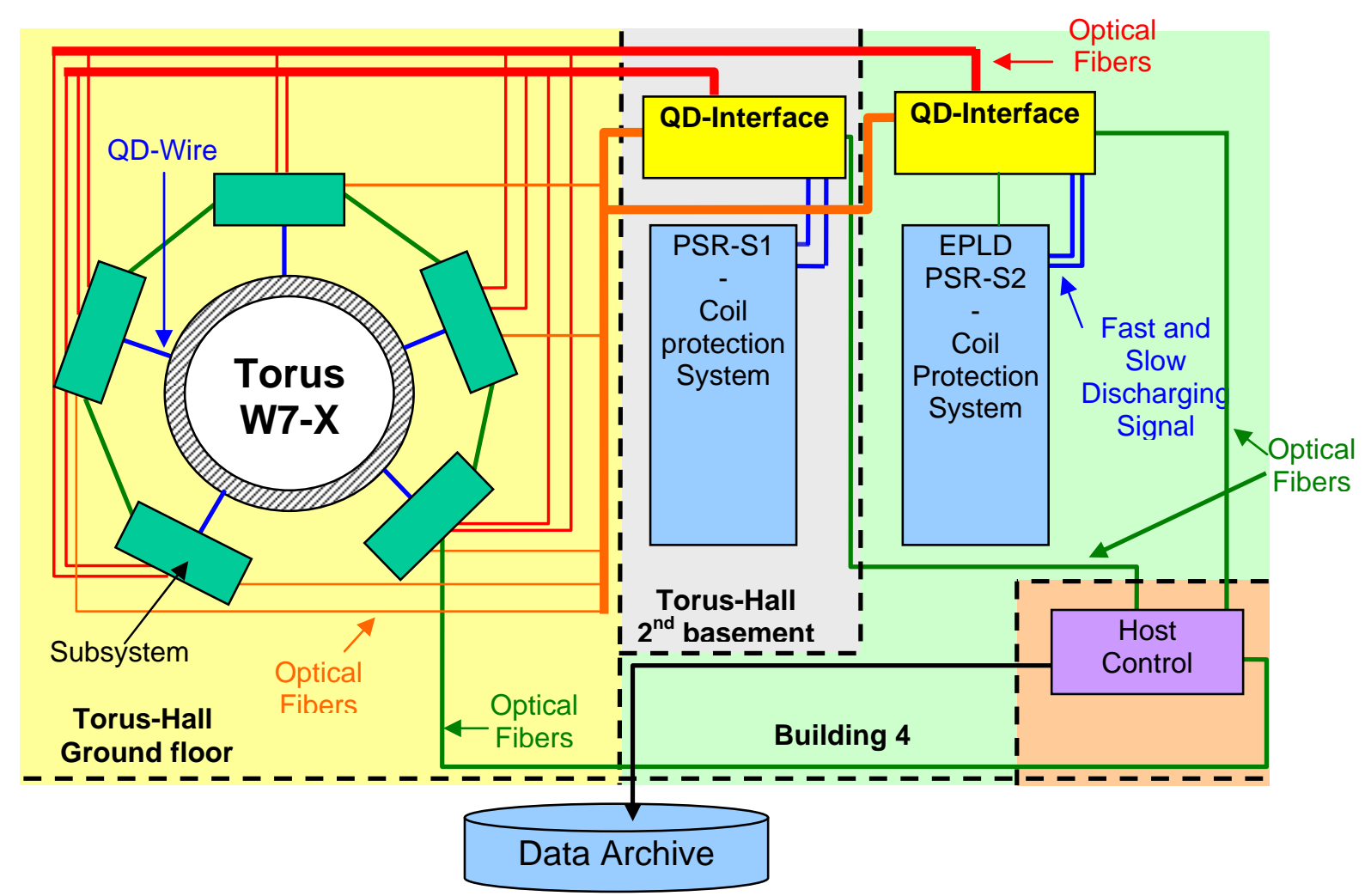

Figure 1: W7-X Quench Detection System. 


\section{Design of Quench Detection Subsystem}

Each QD subsystem contains 72 Quench Detection Units located in 9 QD racks, one DC 24V power supply combined with DC-UPS, one industrial type embedded PC, some electro optical converters and one power cooler with an air water heat exchanger in a closed electric cabinet.

The QD subsystems and the QD interfaces are controlled by the host control. There the system status can be monitored and the QD subsystems as well as the QD interfaces can be parameterized.

All signal paths are designed by optical fibers except for the cables of the fast and slow discharging signals to the host control of the magnet system main power supply.

The embedded PC communicates with each of the Quench Detection Units via two high speed RS 485 interfaces. It allows to configure the Quench Detection Units and the data transfer from the Quench Detection Unit to the embedded PC. The embedded PC of each subsystem is connected with the neighboring subsystems by a fiber optical Ethernet connection. The first of the Quench Detection Subsystems have a fiber optical Ethernet connection to the Quench Detection Host System for control, supervising and configuration of the Quench Detection Units and subsystems.

Each QDU have a special analogue input circuit which was realized as a tunable half bridge front end with different polarity-sensing and limiting functions for suppressing high dynamic voltages. Special filter design is included for RF and noise-suppression and over voltage protection. The QDU are designed with an internal failsafe, programmable self test and redundancy feature, broken wire check of the quench detection cables and connectors inside and outside of the cryostat of W7-X.

The Quench Detection Units acquire and check the differential voltages of the superconducting coils and bus bars continuously. In case of a quench it triggers via the two QD signals the fast discharge of the coils and stores the voltage signals in their internal memory unit. 
The quench signals are in the $\mathrm{mV}$ range and have to be clearly identified within a noisy and a highvoltage background. Each QDU transfers the stored signal dates via one of the two high-speed RS-485 serial interfaces with $20 \mathrm{KV}$ optical isolation barrier to the embedded PC. There will be stored the signal data in the silicon disc inside the PC or an USB stick outside.

Each Quench Detection Unit generates two QD status signals and each QD subsystem combines all these signals to generate two QD status sum signals of one subsystem. These status signals control the QD interfaces, where decided for the right type of discharging of the magnet system.

The figure 2 shows a photo of the first QD subsystem including 72 QDE in 9 racks and the DC power supply $24 \mathrm{~V}$ at the bottom.

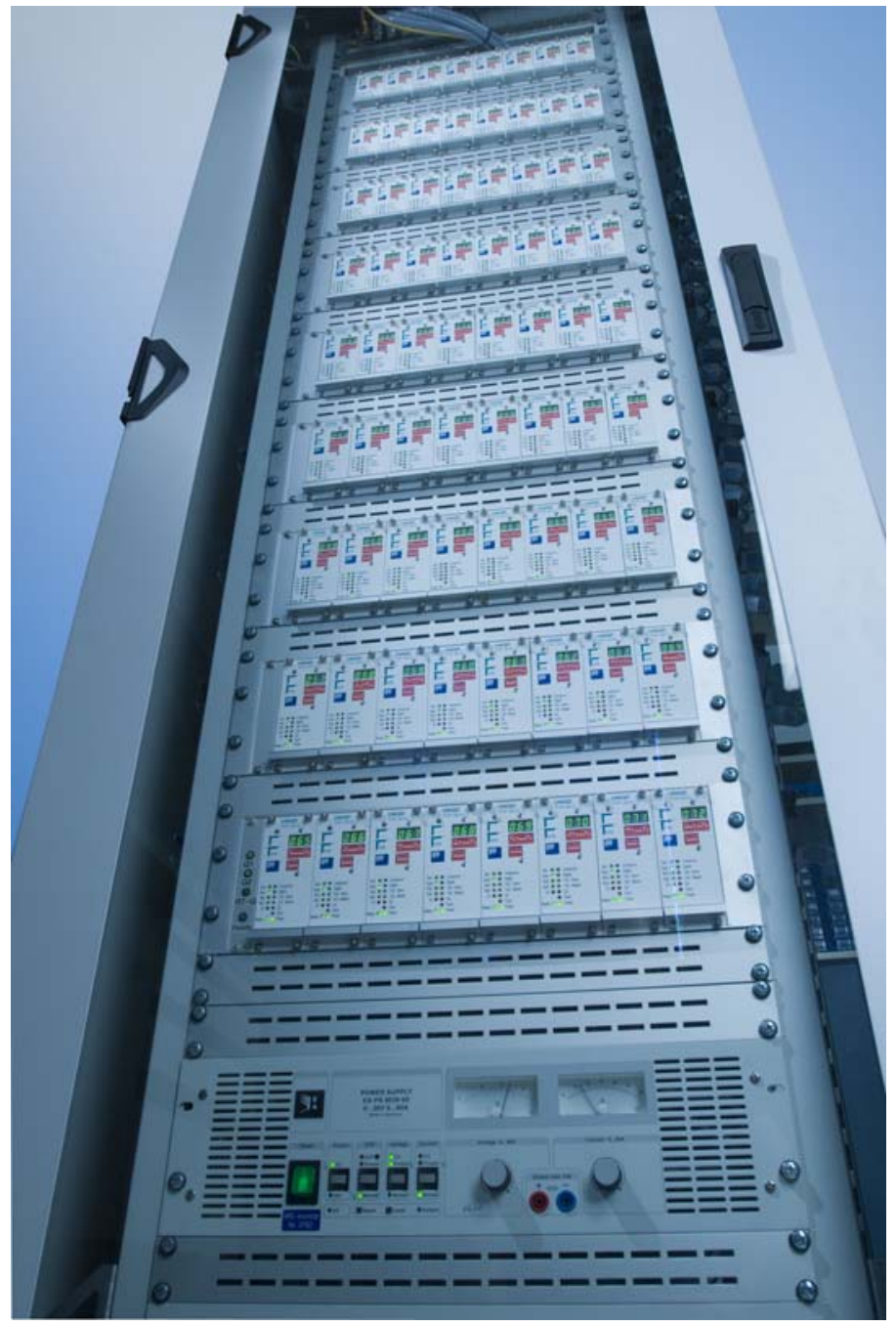

Figure 2: Photo of the Quench Detection Subsystem 


\section{Processing of the Quench Detection Signals}

The present design of the Quench Detection System uses a two-stage safety concept for discharging the magnetic system. This concept includes the possibility two discharging the magnetic system by a fast or slow discharge. The splitting into two discharging processes enabled the dedicated reaction of various events, like quenches and system errors respectively, for protecting the coils and bus bars against damage because of a quench and against the high strain by a fast discharge. In case of a quench the magnetic system a fast discharge has to be performed. But not all system error cases require a fast discharge. In these cases the magnetic system could be discharged by slow discharging. One of these errors could is the failure of the power supply for the subsystem. The internal DC-UPS of the subsystem buffers the power supply for the time of the slow discharge process.

Consequently the Quench Detection System can prioritize the Quench Detection Signals. Each signal is joined of all subsystems at the Quench Detection Interface. By a Boolean logic the signals are estimated and execute a fast or slow discharging depending on the safety level.

The Quench Detection System of W7-X knows two safety levels. The two safety levels are High Safety and Elementary Safety. At High Safety level all signals have the same priority and execute a fast discharge process. At Elementary Safety the signals are divided into two priority-stages. The high stage joins all signals that are quenches and errors, where the Quench Detection System can not guarantees the 100-percent detection capability for a quench, respectively.

Figure 3 descript the signaling concept of the Quench Detection System. First, the QDU detects a quench. In the Single Mode the detector switch's the both signals QDU-1 and QDU-2. The QD subsystem transfers the signals to the QD interface. In both safety levels the QD interface initiate a fast discharge process. 


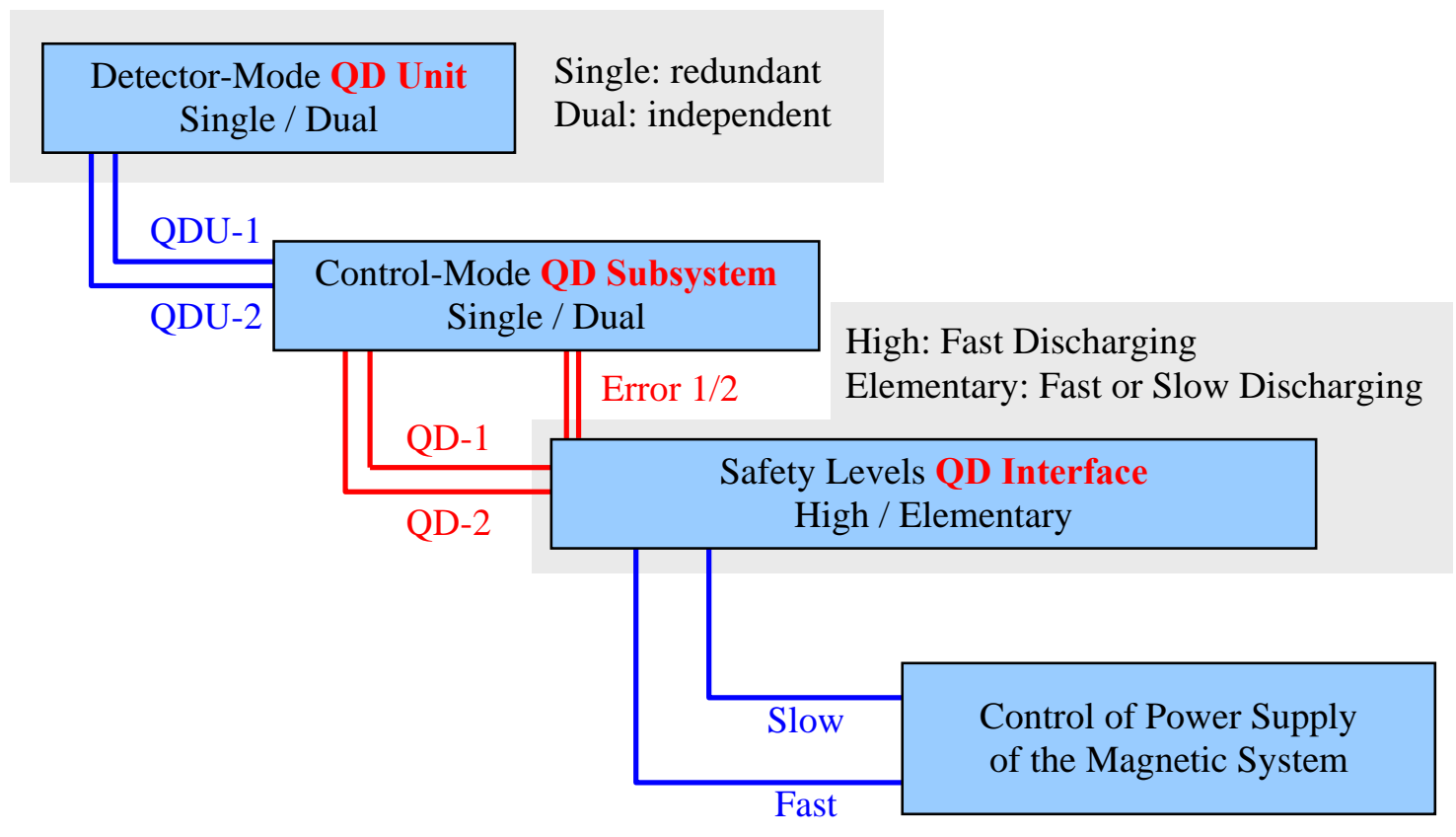

Figure 3: Signaling Concept of the W7-X Quench Detection System.

If the QDU is configured at the Dual Mode the QDU switches only one signal - QDU-1 or QDU-2. At the Dual Mode two different detection levels for a quench can be configured. The QD subsystem transfers the signal to the QD interface. In depend on the safety level the QD interface initiates a fast discharge process at high safety level and a slow discharge process at the elementary safety level.

The third element of signaling concept is the error signals (see Error 1/2). At High Safety level all errors initiate a fast discharging. Error signals, where the Quench Detection System guarantees the 100-percent detection capability for a quench, initiate a slow discharging by Elementary Safety. Errors can be failure of the power supply for the QD subsystems, faulty QDU, wire break of the QD wires or break of optical fibers of signal transmission. 


\section{Design of Quench Detection Interface}

The Quench Detection Interface joins all signals of all QD subsystems. All signals are feeding the decision logic for initiate a fast or slow discharge process in depend on the safety level.

The QD interface is composed of the decision logic by three redundant CPLD units (Complex Programmable Logic Device), a communication unit for transmitting signal states and breaker signals between the QD interface and the host control of the Quench Detection System, a DC-UPS and the optic-electrical converters.

The main part of the QD interface is the three redundant CLPD units. The three units estimate the QD Subsystem signals with a Boolean logic (see Figure 4).

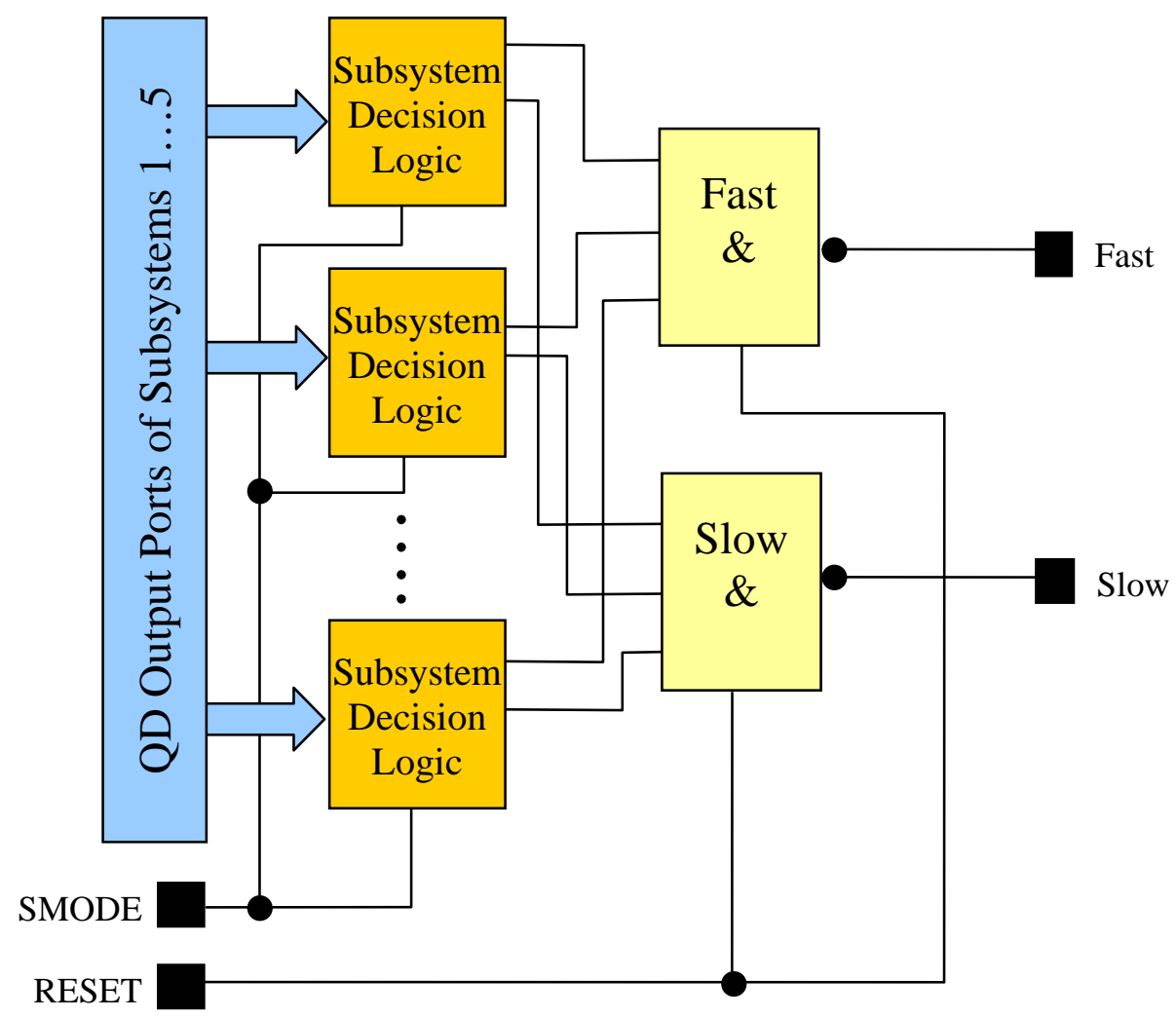

Figure 4: Block diagram of the decision logic of the CPLD. 
Fehler! Verweisquelle konnte nicht gefunden werden.5 shows the Subsystem decision logic with the parallel implemented blocks for the High and Elementary Safety. Both blocks are executed in parallel. The selector of the SMODE (Safety Mode) switches the signals of the High Safety or the Elementary Safety to the outputs Fast and Slow for a fast and slow discharging respectively. With it, both signals Fast and Slow can be independently switched. If a slow discharge process is running and during this time a quench will detected a fast discharging will be initiated. The slow discharge process is immediately changed into the fast discharge process.

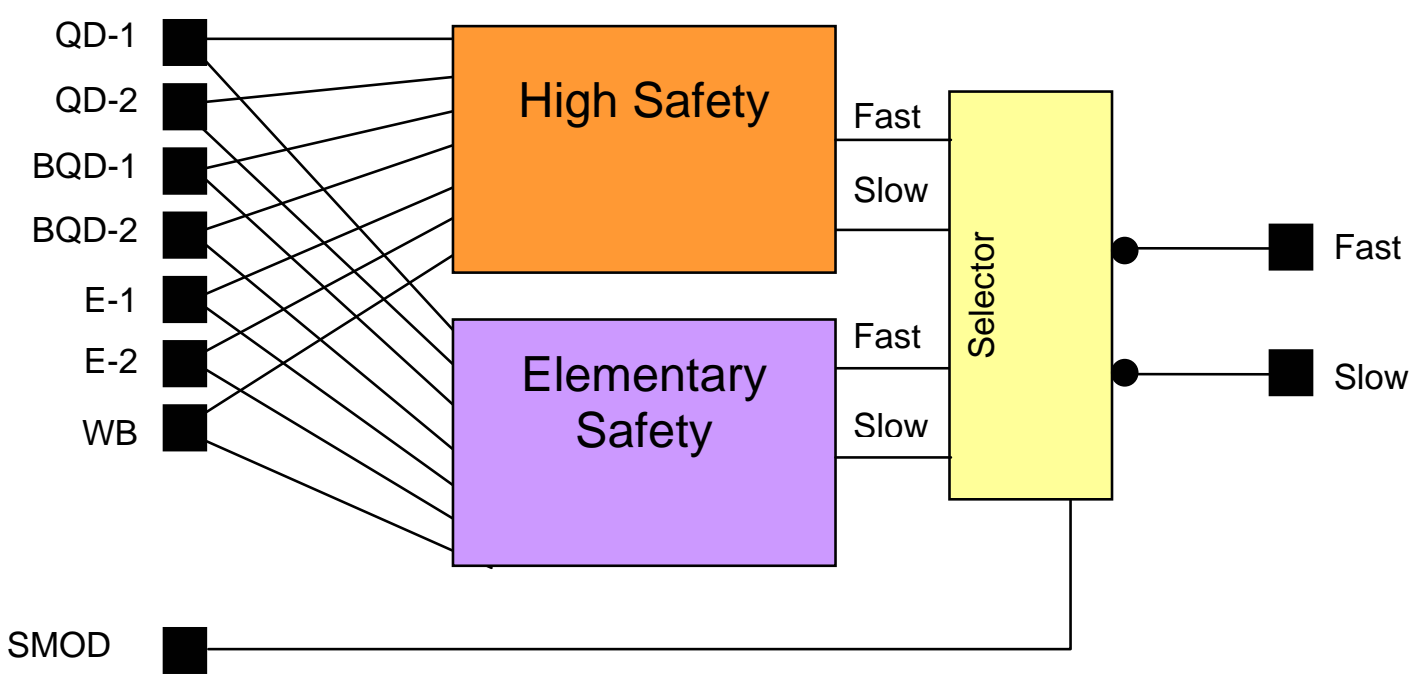

Figure 5: Subsystem decision logic with parallel implemented blocks for High and Elementary Safety.

Figure 6 and Figure 7 shows the block diagram of the logic for High Safety and Elementary Safety. At High Safety level all signals of a QD subsystem are combined by logic AND structure and switch the Fast output. The Slow output is permanently switched to high level and consequently switches to 0. The logic of the Elementary Safety is divided into two ways. One way for the quench signals (QD-1/2) and its wire break signals of the optical fibers (BQD-1/2) as well as the error signals (E-1/2) and the wire break signal of the optical fiber (WB). 


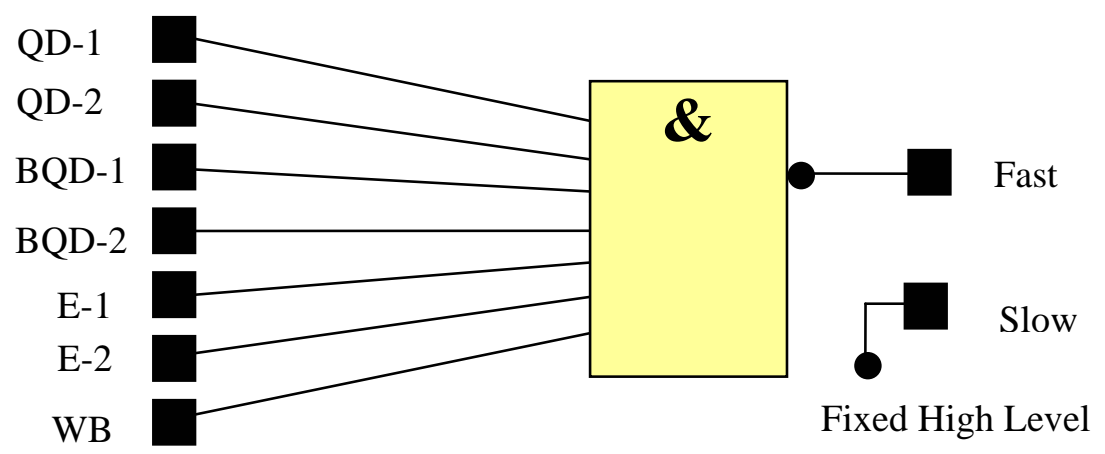

Figure 6: Block diagram of the logic for High Safety.

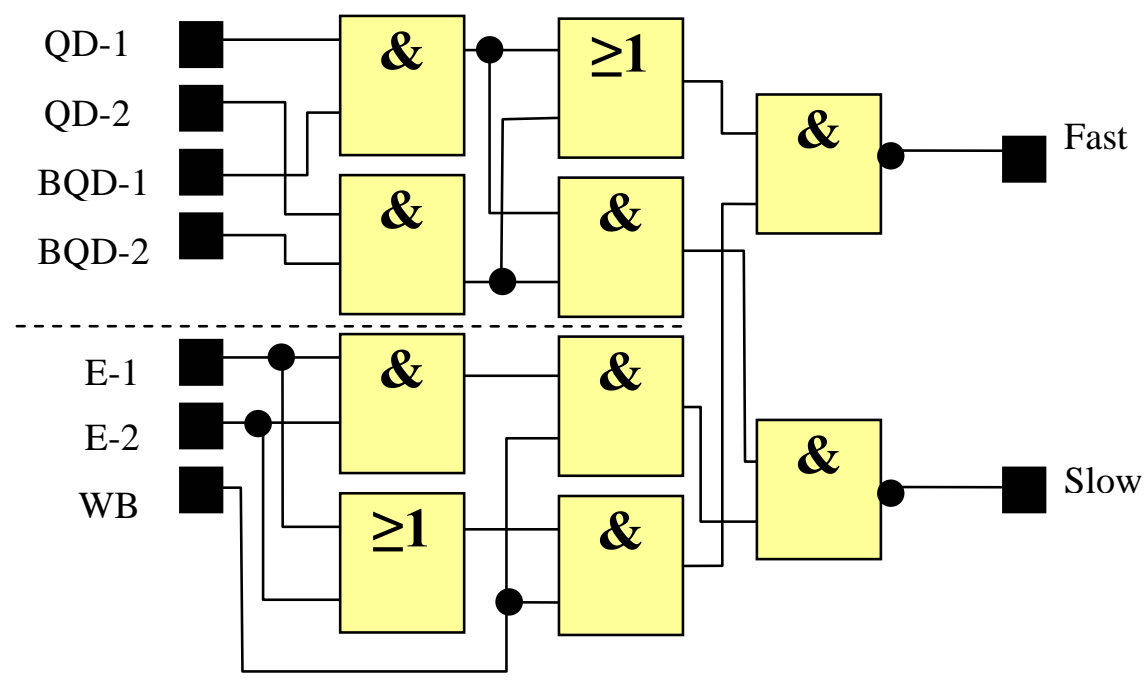

Figure 7: Block diagram of the logic for Elementary Safety.

\begin{tabular}{|c|c|c|}
\hline \multirow[t]{7}{*}{ Legend: } & QD-1: & origin QD Output Signal from QD Unit \\
\hline & QD-2: & redundant QD Output Signal from QD Unit \\
\hline & BQD-1: & origin wire break signal of QD Interface \\
\hline & BQD-2: & redundant wire break signal of QD Interface \\
\hline & E-1: & origin error signal of the QD Subsystem \\
\hline & E-2: & redundant error signal of the QD Subsystem \\
\hline & WB: & break of the fiber optics cables \\
\hline
\end{tabular}


The two-stage safety concept of the W7-X Quench Detection System ensures a minimum of stress of the superconducting components through slow discharging of the magnet system. Otherwise while a critical failure and a quench the QD system will initiate surely a fast discharge.

\section{Conclusions}

This paper has presented the design of the W7-X Quench Detection System consist of QD Subsystems and QD Interfaces processing the QD Signals with the two-stage safety concept using alternatively "High Safety" or "Elementary Safety”. The role of the Boolean Logic of the signal processing and the functionality of the QD Interfaces has been described in detail. The principle of the selection of the safety mode has been explained in this paper. The decision to use high or elementary safety can take by the operating team depended of the current status of the machine only.

\section{References}

M. Borlein: Optimierung eines Quench-Detektions-Systems für supraleitende Magnetspulen Diplomarbeit; Forschungszentrum Karlsruhe; Institut für Technische Physik, 2004

D. Birus et al: Development of Quench Detection System for W7-X

Fusion Engineering and Design 82(2007) p.1400 - 1405

K. Petry: $\quad$ Die neue Quench-Detektion für W7-X

Seminar-Vortrag; 08.07.2008; Forschungszentrum Karlsruhe

Institut für Prozessdatenverarbeitung und Elektronik 